\title{
Aislamiento y caracterización de levaduras presentes en el fruto del Syzygium malaccense (1.) Merr. \&L.M Perry (pomorroso) en la comuna 1 de la ciudad de Neiva- Huila
}

\section{Isolation and characterization of yeasts present in the mountain apple Syzygium malaccense (1.) Merr. \& L.M Perry in commune 1 of Neiva- Huila}

\author{
Lizeth Caterine Trujillo ${ }^{[1]}$ y Sonia Echeverry Hernández ${ }^{[2]}$
}

\begin{abstract}
Resumen
La ciudad de Neiva (Colombia) cuenta con diversidad de frutos tropicales, de los cuales el pomorroso (Syzygium malaccense) es uno de los más abundantes y poco consumido. En este estudio se aislaron y caracterizaron levaduras presentes en el fruto del pomorroso a partir de muestras tomadas en diferentes puntos de la comuna 1 de la ciudad, teniendo en cuenta las épocas de mayor producción al año (junio y diciembre). Para el recuento de UFC/g de fruto se realizaron diluciones seriadas que posteriormente se cultivaron en agar papa dextrosa (PDA) con gentamicina. La caracterización de los diferentes morfotipos se realizó mediante descripción de las colonias en placa y de las células a microscopio. Además, se realizaron pruebas fisiológicas y bioquímicas como la evaluación del crecimiento a $37^{\circ} \mathrm{C}$ y a temperatura ambiente, y la asimilación de algunos azúcares simples. Los recuentos de las levaduras aisladas de las diferentes muestras estuvieron comprendidos entre $10 \times 10^{2}$ y $54 \times 10^{2} \mathrm{UFC} / \mathrm{g}$, a partir de los cuales se identificaron 20 morfotipos diferentes que correspondieron a seis géneros de levaduras: Rhodotorula spp.; Hanseniaspora spp.; Brettanomyces spp.; Candida spp.; Kloekera sp.; Torulopsis sp., además de un hongo dimórfico. La evaluación del medio de cultivo artesanal a base del mismo fruto permitió el crecimiento óptimo de los diferentes morfotipos de levaduras previamente aisladas en PDA; las levaduras aisladas mantuvieron su viabilidad 6 meses después de mantenerse conservadas bajo capa de aceite mineral. Los resultados obtenidos en este estudio pueden ser útiles para la selección de levaduras de importancia en la industria biotecnológica. Además se demuestra que el fruto se encuentra libre de levaduras patógenas.
\end{abstract}

Palabras clave: Syzygium malaccense (pomorroso); levaduras; asimilación de compuestos carbonados; inocuidad de alimentos; medio de cultivo artesanal.

[1] Licenciada en educación básica con énfasis en Ciencias Naturales y Educación ambiental. Universidad Surcolombiana. Neiva-Huila. Av. Pastrana Borrero-Cra 1ª. licatrufi91@hotmail.com

[2] M.Sc. Microbiología. Docente asistente. Tutora semillero de investigación Virhobac. Neiva-Huila. Av. Pastrana BorreroCra $1^{\text {a }}$.sonia.echeverry@usco.edu.co

Recibido: 29 abril 2015 • Aceptado: 28 mayo 2015 


\begin{abstract}
The city of Neiva (Colombia), hosts a diversity of tropical fruit, including the rose apple (Syzygium malaccense), which is one of the most abundant, but rarely consumed. In this study, the yeasts in the rose apple were isolated and characterized after samples were taken from different areas of the city's Comuna 1, taking into account that they are most abundant in June and December. In order to calculate the CFU/g of the fruit, serial dilutions were undertaken that were subsequently cultivated in Potato Dextrose Agar (PDA) with gentamicin. The characterization of the different morphotypes is carried out through using the plate count method and analyzing the cells through a microscope. Moreover, physiological and biochemical tests were undertaken, such as the evaluation of bacterial growth at a temperature of $37^{\circ} \mathrm{C}$ and at room temperature, and the assimilation of some simple sugars. The calculations of the isolated yeasts from the different samples were found to be between $10 \times 10^{2}$ and $54 \times 10^{2} \mathrm{CFU} / \mathrm{g}$. Twenty different morphotypes were identified, which were understood to belong to six different genera of yeast: Rhodotorula spp.; Hanseniaspora spp; Brettanomyces spp; Candida spp; Kloeckera; Torulopsis, as well as a Dimorphic fungus. The evaluation of the artisanal cultivation method for the same fruit allowed for the optimum increase of the different yeast morphotypes that were previously isolated in the PDA. The isolated yeasts kept their viability for six months conserved under a mineral-oil overlay. The results obtained in this study can be used for the selection of important yeasts in the bio-technic industry. Furthermore, it is shown that the fruit is free of pathogenic yeast.
\end{abstract}

Key words: Syzygium malaccense (pomarrosa); yeasts; assimilation of carbon compounds; food safety; artesanal cultivation method.

\section{Introducción}

La biodiversidad fúngica es considerada una de las principales fuentes de innovación biotecnológica y una gran variedad de hábitats han sido seleccionados con el fin de encontrar microorganismos biotecnológicamente importantes (Bhadra et al. 2008).

Las levaduras que habitan los frutos, gracias a los múltiples mecanismos de adaptación que han desarrollado frente a condiciones adversas, como la exposición directa a los rayos solares, periodos de desecación, temperaturas y condiciones de osmolaridad extrema, pueden ser utilizadas en varios campos industriales y biotecnológicos (Uribe 2007). Las condiciones que prevalecen en estos hábitats naturales determinan la actividad metabólica, el crecimiento y la supervivencia de dichas levaduras (Deak, 2006).

La exploración de la biodiversidad de las levaduras de ambientes naturales extremos puede aportar datos valiosos referidos al papel de las mismas en esos ambientes y a sus mecanismos de adaptación (Libkind, 2005). La habilidad de adherencia a la superficie de frutos y hojas que han desarrollado algunas levaduras les ha permitido sobrevivir a ambientes con alta exposición a la radiación solar por medio de la producción de pigmentos oscuros, que a su vez, les permite proveer una protección natural a la planta contra los diferentes patógenos (Lindow, 2004).
En Colombia, el pomorroso como fruta no es tan conocido, ya que no se comercializa ni cultiva de la misma forma que las demás frutas tropicales. Sin embargo, es utilizado en algunas ciudades de nuestro país como alimento, ya sea en fresco por su exótico sabor o elaborado en postres, jaleas y dulces debido a su contenido nutricional (Medina y Calderón, 2008). Pese a las propiedades de inocuidad que posee el fruto del pomorroso, la comunidad neivana ha creado un mito acerca de su consumo refiriendo malestar al momento de ingerirlo por la posible presencia de microorganismos.

Teniendo en cuenta la abundancia del fruto y la poca investigación a nivel microbiológico que se ha realizado en la región sobre el mismo, el objetivo de este trabajo es aislar y caracterizar las levaduras presentes en este tipo de frutos tropicales que posteriormente pueden ser utilizadas con fines biotecnológicos en la producción de vino o como agente biocontrolador, así como la evaluación de un método viable de conservación para levaduras y de un medio de cultivo natural a base del mismo fruto.

\section{Materiales y métodos}

Los muestreos fueron realizados en la comuna 1 , ubicada al noroccidente de la ciudad de Neiva, con una 
extensión de $2.4 \mathrm{Km}^{2}$ y comprendida por 43 barrios reconocidos por el Departamento de Planeación $\mathrm{Mu}$ nicipal. Esta comuna cuenta con una población aproximada de 43.750 habitantes.

Para la recolección del material de estudio se empleó el método de muestreo sistemático en grillas, con un tamaño de muestra de 160 árboles distribuidos en 17 grillas. Se realizaron 10 muestreos comprendidos entre enero y junio de 2014, teniendo en cuenta las dos épocas de cosecha anual del árbol: la primera, entre diciembre y enero y la segunda entre junio y julio de cada año. Las muestras (una muestra corresponde a un fruto en estado apropiado de maduración) fueron empacadas y transportadas individualmente bajo condiciones asépticas en bolsas plásticas tipo ziploc; luego se procesaron inmediatamente en el laboratorio.

El procesamiento de muestras se realizó según el protocolo adaptado del Manual de Técnicas Básicas de Microbiología de Alimentos de la Universidad Nacional Autónoma de México. Este consistió en homogeneizar en licuadora los frutos en $90 \mathrm{ml}$ de agua peptonada estéril y a partir de ella transferir $1 \mathrm{ml}$ a un tubo que contenía $9 \mathrm{ml}$ del mismo diluyente; de cada una de las diluciones se sembraron por duplicado 100 $\mu \mathrm{L}$ de la muestra en agar papa dextrosa (PDA) con gentamicina y se incubó a temperatura ambiente de 3 a 5 días; para el recuento de colonias se empleó el método de recuento estándar para mohos y levaduras según la Norma Técnica Colombiana (NTC) 4092.

En la descripción de la morfología de colonia se tuvo en cuenta la forma, elevación, borde, consistencia y color y mediante tinción de Gram se realizó la descripción de la morfología celular teniendo en cuenta la forma, reproducción y estructuras típicas como la presencia de pseudohifas.

De los morfotipos descritos se aislaron por duplicado cultivos puros en PDA para determinar la asimilación de azúcares mediante la técnica de auxonograma; se evaluaron sacarosa, glucosa, maltosa, galactosa, lactosa, arabinosa como únicas fuentes de carbono y nitrato de sodio como única fuente de nitrógeno, comparado con la prueba de fermentación en agar TSI. Además, se evaluó el crecimiento a temperatura ambiente y a $37^{\circ} \mathrm{C}$.

La viabilidad de las levaduras se probó con base en la estabilidad morfológica de la colonia y celular, en las pruebas de asimilación de azúcares y de nitrógeno y en el crecimiento a temperatura ambiente y $37^{\circ} \mathrm{C}, 6$ meses después de permanecer bajo capa de aceite mineral.

Se preparó un medio de cultivo artesanal a base del extracto del pomorroso como única fuente de nutrientes y como agente solidificante gelatina sin sabor, adicionando como inhibidor del crecimiento bacteriano $1 \mathrm{ml}$ de gentamicina a una concentración de $0,24 \mathrm{mg} / \mathrm{ml}$.

\section{Resultados y discusión}

En la tabla 1 se presentan los resultados del recuento de UFC/g de muestra y el total de morfotipos aislados, según la época de muestreo. De acuerdo con Cano (2006), un recuento bajo de levaduras no asegura la ausencia de patógenos o sus toxinas; de la misma manera, un recuento elevado no significa presencia de microorganismos patógenos.

Sin embargo, para poder establecer la inocuidad del fruto del pomorroso es necesario determinar la especie de las levaduras encontradas, ya que algunas son propias del fruto y a la vez son responsables de sus características organolépticas.

En la tabla 2 se relaciona la pigmentación de las colonias con la temporada de muestreo. Como lo indica Medina y Calderón (2008) la especie del Syzygium malaccense posee una ramificación simpodial y una disposición caulinar del fruto dentro de las ramas del árbol, lo cual condiciona la prevalencia de las colonias color blanco, tanto en la temporada seca como lluviosa.

Tabla 1. Recuento de UFC por época de producción del árbol (NTC 4092)

\begin{tabular}{lcccccccccc}
\hline $\mathbf{N}^{\circ}$ de muestreo & M1 & M2 & M3 & M4 & M5 & M6 & M7 & M8 & M9 & M10 \\
\hline Época de muestreo & Enero & Enero & Febrero Febrero Febrero & Mayo & Mayo & Mayo & Mayo & Junio \\
UFC/g & $31 * 10^{2}$ & 0 & 0 & 0 & $10 * 10^{2}$ & 0 & $18^{*} 10^{2}$ & $24 * 10^{2}$ & $54^{*} 10^{2}$ & $11^{*} 10^{2}$ \\
Morfotipos caracterizados & 2 & 0 & 0 & 0 & 5 & 0 & 4 & 1 & 6 & 2 \\
\hline
\end{tabular}


Tabla 2. Pigmentación de la colonia según la temporada de muestreo

\begin{tabular}{lcc}
\hline Temporada de muestreo & Color de la colonia & Número de morfotipos \\
\hline Seca (meses de enero y febrero) & Blanco & 5 \\
& Salmón & 1 \\
& Coral & 1 \\
\hline Lluviosa (meses de mayo y junio) & Blanco & 8 \\
& Coral & 2 \\
& Beige & 1 \\
& Marrón & 1 \\
\hline
\end{tabular}

Resultados similares fueron obtenidos por Usman (2012), quien reportó que la mayoría de las colonias levaduriformes aisladas de la pulpa del pomorroso presentaron color blanco (20) y beige (18). De igual manera, Uribe (2007) reportó que las colonias aisladas de la filósfera de la mora presentaron coloración blanco (23), crema (17) y rosa (6).

Para el caso de las colonias que presentaron pigmentación, esta caracteristica está asociada a la resistencia que muchas levaduras han desarrollado para sobrevivir a ambientes con alta exposición a la radiación solar (Lindow, 2004), al igual que la capacidad para realizar cambios estructurales al interior de la célula como respuesta a un cambio brusco generado por estrés osmótico (Mager, 2002).

Algunas de las levaduras aisladas presentaron cambios en su morfología de colonia. Tal como se muestra en la tabla 3, el morfotipo 8, además de presentar una variación en su pigmentación pasando de color beige con centro más oscuro a gris perlado con pigmentos difusibles a la colonia color beige, presen- tó pigmentos de colonia difusibles al medio color mandarina. Como lo indica Dastager et al., 2006 los pigmentos difusibles son producidos principalmente durante la fase estacionaria de la curva de crecimiento de los hongos, donde llevan a cabo su metabolismo secundario sintetizando metabolitos volátiles, compuestos aromáticos y quinonas que pueden tener efecto antibiótico.

Se identificaron 20 morfotipos diferentes que correspondieron a seis géneros de levaduras: Rhodotorula spp.; Hanseniaspora spp.; Brettanomyces spp.; Candida spp.; Kloekera spp.; Torulopsis spp.

El morfotipo 10 presentó una variación en su morofología, pasando de una apariencia típica de un hongo levaduriforme a la de un hongo filamentoso, tal como se indica en la figura 1.A. Lo anterior sugiere el desarrollo de un hongo dematiáceo con una fase levaduriforme y una fase micelial la cual suele confundirse con frecuencia con especies del género Aspergillus (figura 1.B) y que corresponde a lo encontrado en esta investigación (Cecil, 1996).
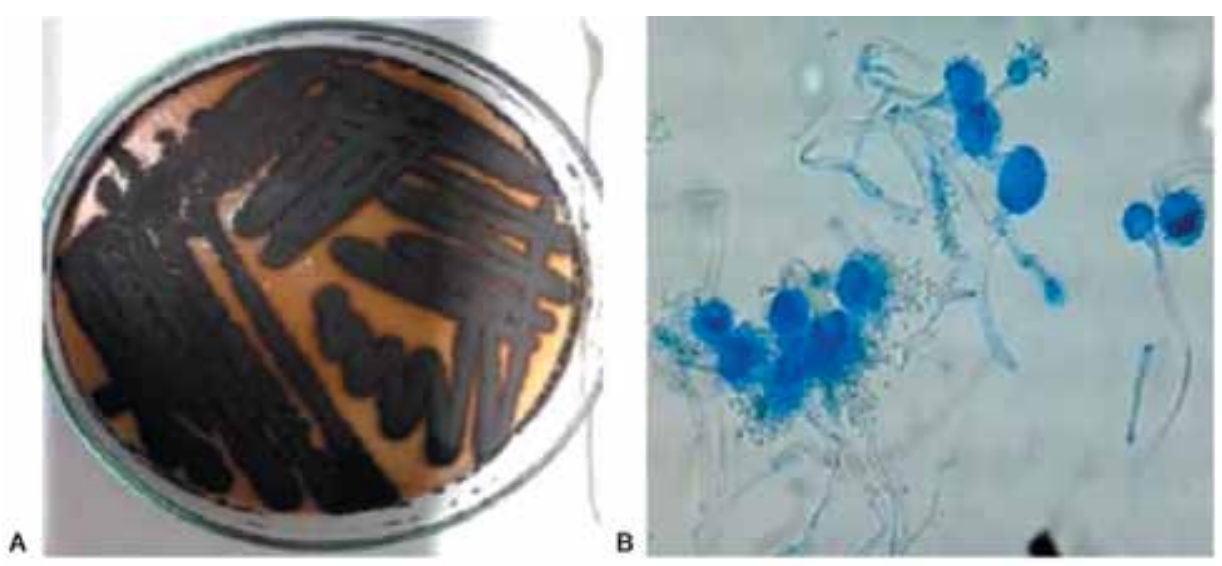

Figura 1. A. Morfología de colonia con crecimiento en PDA. B. Morfología celular. 
Tabla 3. Descipción de la morfología de colonia y morfología celular de los morfotipos aislados.

\begin{tabular}{lll}
\hline Morfotipo & Descripción de colonia & Descripción celular \\
\hline
\end{tabular}

1 Colonia circular de $4 \mathrm{~mm}$ de diámetro, borde entero,

Levadura elíptica, gemante, con un solo brote de elevación convexa, superficie brillante, consistengemación en el ápice y escasa pseudohifa. cia cremosa, color salmón, con centro rosado y de morfología compleja, borde traslúcido.

$4 \quad$ Colonia circular de $11 \mathrm{~mm}$ de diámetro, borde entero, elevación umbonada, superficie serosa, consistencia cremosa, color blanco con centro crema. Con olor característico a fermentación.

8

Colonia circular de 3mm de diámetro, borde entero, elevación convexa, superficie brillante, consistencia cremosa, color beige con centro más oscuro. Pasados tres días de crecimiento, presenta pliegues y centro con gránulos color gris, pigmentos difusibles al medio color mandarina; el repique es de color gris perlado con puntos color beige y presenta pigmentos difusibles al medio color mandarina.
Levadura ovoide, algunas de gran tamaño, con abundante gemación en el ápice y escasa gemación lateral, con un solo brote de gemación y pseudohifa.

Levadura elíptica, gemante con un solo brote de gemación en el ápice, con escasa pseudohifa.

10 Colonia circular de $4 \mathrm{~mm}$ de diámetro, borde entero, plana, superficie cerosa, consistencia pétrea, crecimiento concéntrico, color marrón oscuro, con olor

Levadura ovoide, gemante con un solo brote de gemación en el ápice, con pseudohifa.

característico a fermentación.

11 Colonia circular de 2,5 mm de diámetro, borde ente-

Levadura elíptica, muy pocas gemantes con un solo ro, elevación cóncava, superficie brillante, consistencia cremosa, color blanco. Pasadosalgunos días brote en el ápice, con abundante pseudomicelio. de incubación, la colonia se torna color beige.

Colonia irregular de 4 mm de diámetro, borde escarolado, elevación pulvinada, superficie cerosa, consistencia cremosa, color rosado, toda la colonia presenta pliegues. Pasados días de incubación la colonia se torna color salmón.
Levadura elíptica de mayor longitud, con escasa gemación y un solo brote en el ápice, con abundante pseudohifa.
17 Colonia circular de $2 \mathrm{~mm}$ de diámetro, borde entero, elevación convexa, superficie brillante, consistencia cremosa, color blanco, con olor característico a fermentación.
Levadura ovoide en gemación con un solo brote en el ápice, algunas pocas con gemación multilateral, con escasa pseudohifa.
Colonia circular de $8 \mathrm{~mm}$ de diámetro, borde poco ondulado, elevación convexa, superficie cerosa, consistencia cremosa, color blanco con centro crema, con olor característico a fermentación.
Levadura elíptica alargada y gruesa, pocas en gemación polar y escasamente gemación lateral con un solo brote. Dispuestas mayormente en parejas y tétradas. 


\section{Caracterización fisiológica de los morofotipos aislados}

Mediante caracterización fisiológica se determinó que los morfotipos aislados presentan un mejor desarrollo a temperatura ambiente $\left(24\right.$ a $\left.27^{\circ} \mathrm{C}\right)$ (Tabla 4), lo cual corresponde a lo reportado por Uribe (2007) que al evaluar el efecto de la temperatura sobre el crecimiento de las levaduras aisladas de la filósfera de la mora, el rango óptimo de crecimiento se presentó entre 25 a $37^{\circ} \mathrm{C}$.

En la tabla 4 se muestran los resultados de la caracterización bioquímica mediante la evaluación de la asimilación de azúcares (auxonograma), observándose mayor crecimiento de levaduras alrededor de los sensidiscos impregnados con galactosa (18) y glucosa (15); el menor crecimiento se observó alrededor de los sensidiscos con maltosa (7). La asimilación de nitrato de sodio resultó negativa para la mayoría de morfotipos.

Teniendo en cuenta tanto la caracterización morfológica de colonia como celular y los resultados de las pruebas fisiológicas y bioquímicas, se identificaron seis (6) géneros de levaduras de acuerdo con el uso de claves dicotómicas; estos grupos correspondieron a Rhodotorula spp. (6 morfotipos); Hanseniaspora spp. (3 morfotipos); Brettanomyces spp. (2 morfotipos); Candida spp. (2 morfotipos); Kloekera sp. (1 morfotipo); Torulopsis sp. (1 morfotipo); hongos dimórficos (1 morfotipo) y 4 morfotipos a los que no se identificó el género. Los resultados de las levadu- ras aisladas en la comuna 1 de la ciudad de Neiva a partir de la pulpa y corteza del fruto de pomorroso muestran correspondencia con las aisladas de la pulpa de pomorroso en la Universidad del Valle, donde se identificaron 8 especies diferentes de los géneros Hanseniaspora, Pichia, Sporidiobolus,y Rhodotorula, Candida Clavispora, especies biotecnológicamente importantes.

Especies como Candida guillermondii aislada de frutos de uchuva han sido estudiadas por Buitrago y Escobar (2009) como una alternativa viable para retardar la pudrición que presenta el fruto del banano. Uribe (2007), en su estudio de caracterización fisiológica de levaduras aisladas de la filósfera de la mora encontró especies identificadas como Candida kunwiensis, Cryptococcus laurentii, Cryptococcus humicola, Dipodoscus tetrasperma, que mostraron el mejor comportamiento en las pruebas de crecimiento a diferentes temperaturas, $\mathrm{pH}$ y altas concetraciones de glucosa.

Teniendo en cuenta los resultados de los estudios anteriores y los de esta investigación en particular, la presencia del género Candida en el fruto de pomorroso puede constituirse en una fuente de estudio para posibles métodos de control biológico en cultivos de la región surcolombiana.

\section{Elaboración del medio de cultivo artesanal}

En medio de cultivo artesanal a base de pomorroso (figura 2A) e inoculadas las levaduras aisladas de este

Tabla 4. Resultados del auxonograma y del crecimiento a diferentes temperaturas

\begin{tabular}{|c|c|c|c|c|c|c|c|c|c|c|c|c|c|c|c|c|c|c|c|c|}
\hline \multirow{2}{*}{ Prueba } & \multicolumn{20}{|c|}{ Morfotipo } \\
\hline & \multirow{2}{*}{$\begin{array}{r}\text { M1 } \\
-\end{array}$} & \multirow{2}{*}{$\begin{array}{c}\text { M2 } \\
-\end{array}$} & \multirow{2}{*}{$\begin{array}{c}\text { M3 } \\
-\end{array}$} & \multirow{2}{*}{$\begin{array}{c}\text { M4 } \\
+ \\
+\end{array}$} & \multirow{2}{*}{$\begin{array}{c}\text { M5 } \\
-\end{array}$} & \multirow{2}{*}{$\begin{array}{c}\text { M6 } \\
-\end{array}$} & \multirow{2}{*}{$\begin{array}{c}\text { M7 } \\
\text { R }\end{array}$} & \multirow{2}{*}{$\frac{M 8}{\mathrm{R}}$} & \multirow{2}{*}{$\frac{\mathrm{Mg}}{\mathrm{R}}$} & \multirow{2}{*}{$\begin{array}{r}\mathrm{M} 10 \\
\mathrm{R}\end{array}$} & \multicolumn{4}{|c|}{ M11 M12 M13 M14 } & \multicolumn{2}{|c|}{ M15M16 } & \multicolumn{4}{|c|}{ M17 M18 M19 M20 } \\
\hline Crecimiento a $37^{\circ} \mathrm{C}$ & & & & & & & & & & & $\mathrm{R}$ & - & + & - & - & - & + & + & - & + \\
\hline Crecimiento a $\mathrm{T}^{\circ}$ ambiente $\left(28^{\circ} \mathrm{C}\right)$ & + & + & + & + & + & + & + & + & + & + & + & + & + & + & + & $\mathrm{R}$ & + & + & + & + \\
\hline \multicolumn{21}{|l|}{ Asimilación de comp. Carbonados } \\
\hline Sacarosa & - & + & + & - & + & + & - & - & + & + & + & + & - & + & - & - & - & - & + & - \\
\hline Glucosa & + & - & + & + & + & + & - & + & + & + & + & + & + & + & - & + & + & + & - & - \\
\hline Maltosa & - & - & + & - & + & - & - & + & - & + & - & - & - & - & + & - & - & + & + & - \\
\hline Galactosa & + & + & + & + & + & + & + & + & + & + & + & + & + & - & + & + & - & + & + & + \\
\hline Lactosa & + & + & - & + & - & - & + & - & + & + & + & - & - & + & + & + & + & - & + & + \\
\hline Arabinosa & - & - & + & + & + & - & - & + & + & + & - & + & + & + & - & - & + & + & - & + \\
\hline asimilación de comp. Nitrogenados & & & & & & & & & & & & & & & & & & & & \\
\hline Nitrato de sodio & $*$ & $*$ & - & $*$ & - & $*$ & - & $*$ & - & $*$ & - & $*$ & - & - & - & - & - & - & - & - \\
\hline
\end{tabular}

+ positivo - negativo $\mathbf{R}$ regular * sin determinar 
fruto y desarrolladas previamente en el agar papa dextrosa (PDA) crecieron a los 3 días de cultivo y a temperatura ambiente $\left(24\right.$ a $27^{\circ} \mathrm{C}$ ) (figura 2B). En comparación con el PDA, el crecimiento de las levaduras en el medio de cultivo artesanal fue más rápido, este resultado probablemente se deba a que las levaduras se adaptan más fácilmente a los medios de cultivo naturales.

Resultados similares fueron expuestos por Buitrago y Escobar (2009) quienes elaboraron un agar banano amarillo y verde, en donde se evidenció un buen crecimiento de la levadura Candida guillermondii en ambas concentraciones de disponibilidad de nutrientes (agar banano amarillo y agar banano verde).

De igual manera para comprobar las propiedades nutricionales del medio de cultivo artesanal, se sembraron cepas de hongos filamentosos los cuales se aislaron de frutas y verduras (figura 2C). A las 24 horas de cultivo a temperatura ambiente $\left(24\right.$ a $\left.27^{\circ} \mathrm{C}\right)$ se observó buen crecimiento de colonias, lo cual permite demostrar que el medio de cultivo artesanal a base de pomorroso puede ser utilizado como medio de cultivo para una gran variedad de microorganismos fúngicos que no licúan la gelatina.

El medio de cultivo artesanal a base de pomorroso (MAP) debido a sus características particulares como su apariencia y consistencia, requiere de precaución al momento de ser elaborado y utilizado: no resiste la incubación a los $37^{\circ} \mathrm{C}$ (se funde), se conserva invertido a temperatura ambiente $\left(24\right.$ a $27^{\circ} \mathrm{C}$ ) y, aunque la superficie del agar no permite realizar una buena siembra debido a su consistencia antideslizante y pegajosa, si permite el buen desarrollo y crecimiento de organismos fúngicos.

\section{Evaluación del aceite mineral como método de conservación}

Los 20 morfotipos caracterizados en este estudio presentaron viabilidad al ser conservados durante 6 meses en capa de aceite mineral, ya que al realizar las pruebas de asimilación de azúcares, nitrato $\left(\mathrm{NaNO}_{3}\right)$ y crecimiento a diferentes temperaturas (temperatura ambiente: 24 a $27^{\circ} \mathrm{C} \mathrm{y} 37^{\circ} \mathrm{C}$ ), no se alteraron sus características morfológicas y fisiológicas.

Lo anterior es confirmado por Panizo et al. (2005) quienes utilizaron 411 hongos: 170 levaduras y 241 filamentosos, escogidos al azar de la Micoteca del Departamento de Micología del Instituto Nacional de Higiene Rafael Rangel ubicada en Venezuela y los preservaron por duplicado tanto en agua destilada como bajo capa de aceite mineral a temperatura promedio de $8^{\circ} \mathrm{C}$. De las 170 levaduras estudiadas, el $100 \%$ se mantuvieron puras, viables y estables morfológicamente por los dos métodos de preservación usados, variando el tiempo de preservación entre 2 a 48 años, siempre y cuando se subcultivaran en promedio cada año y medio.

\section{Conclusiones}

En el fruto de Syzygium malaccense no se aislaron levaduras patógenas, los géneros que se caracterizaron e identificaron fenotípica y bioquímicamente correspondieron a: Hanseniaspora spp., Brettanomyces spp., Rhodotorula spp. Candida spp., Kloekera spp. y Torulopsis spp., además de hongos dimórficos y 4 morfotipos a los que no se identificó el género. Estos aislados de levaduras pueden ser biotecnológicamente importantes tanto para la industria vinícola como para el sector agropecuario de la región.
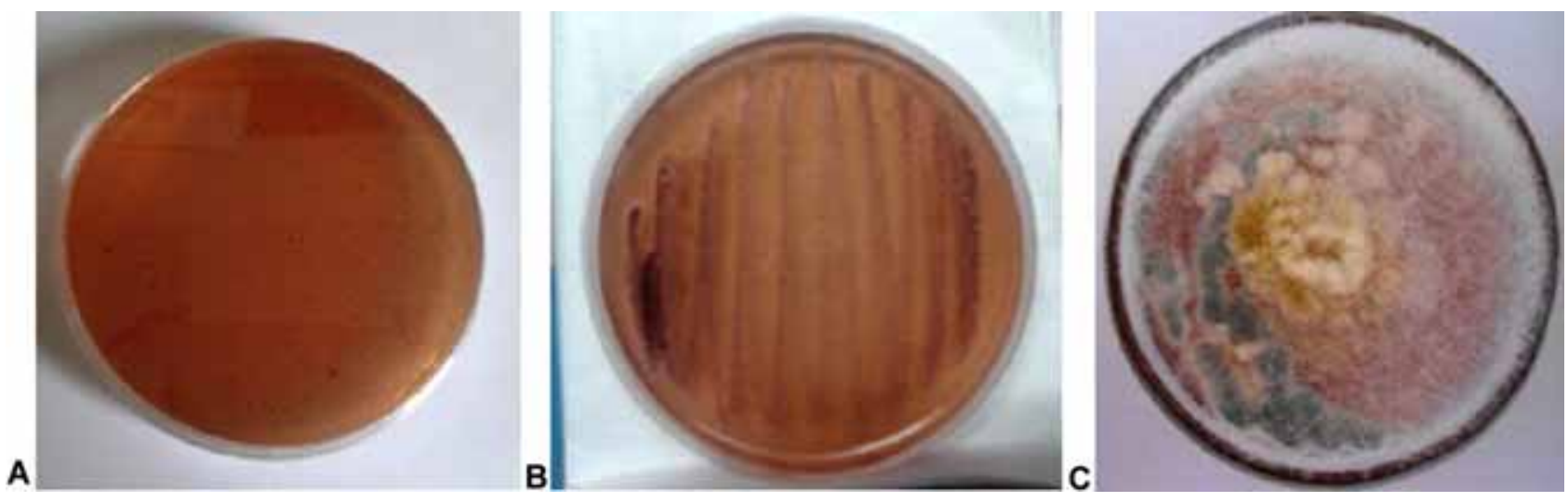

Figura 2. A.MAP. B. Colonias levaduriformes. C. Hongos filamentosos. 
El método de conservación en aceite mineral es recomendable para la implementación de colecciones biológicas, ya que permite mantener la viabilidad de los microorganismos levaduriformes, siempre y cuando se encuentre en constante vigilancia.

El MAP puede ser utilizado como medio de cultivo para una gran variedad de microorganismos fúngicos, ya que permitió aislar tanto las levaduras presentes en el fruto como hongos filamentosos tomados de otros alimentos (frutas y verduras).

\section{Referencias bibliográficas}

1. Ángel, D. 2006. Evaluación de técnicas de conservación para hongos filamentosos y levaduriformes en el cepario de la Pontificia Universidad Javeriana. Trabajo de grado Microbiología Industrial. Pontificia Universidad Javeriana. Facultad de Ciencias. Bogotá, D.C.

2. Barreto, I. Castro, C. 2011.Cuantificación de la actividad enzimática tipo quitinasa de actinomicetos y su capacidad antagónica frente a hongos fitopatógenos. Trabajo de grado Microbiología Industrial. Bogotá D.C. Pontificia Universidad Javeriana. Facultad de Ciencias.

3. Bhadra, B., Rao, R., Singh, P., Sarkar, P., Shivaji, S. 2008. Yeasts and Yeast-Like Fungi Associated with Tree Bark: Diversity and Identification of Yeasts Producing Extracellular Endoxylanases. Curr Microbiol 56:489-494.

4. Buitrago, J., Escobar, M. 2009. Aplicación de levadura Candida spp. como una alternativa viable para la retardación en la pudrición del banano (Musa acuminata). Trabajo de grado Microbiología Industrial. Pontificia Universidad Javeriana. Facultad de Ciencias. Bogotá D.C.

5. Camacho, A., Giles, M., Ortegón, A., Palao, M., Serrano, B., Velázquez, O. 2011. Método para la cuenta de mohos y levaduras en alimentos. Manual de Técnicas Básicas de Microbiología de alimentos. Facultad de Química, Universidad Autónoma de México.

6. Casal, J., Mateu, E. 2003. Tipos de muestreo. Revista de Epidemiología y Medicina Preventiva Veterinaria. [Online]. n 1 [citado 2013-07-06], pp. 3-7. Disponible en: <http://www.scielo.org.co/scielo.php? script=sci_arttext\&pid=S0301- 73252010000 200001\&lng=pt\&nrm=iso>. ISSN0301-7325.
7. Cecil. 1996. Tratado de medicina interna. Vol. 3,20a ed. Bennett, C. y Plum, F. Sounders Company, Phyladelphia.

8. Deak, T. 2006. Environmental factors influencing Yeasts. In ROSA, C. \& G. PÉTER, (eds.), The yeast Handboock: Biodiversity and Ecophysiology of Yeasts, pp. 155-174. Springer-Verlag, Berlin, Heidelberg, New York.

9. Dastager, S. Li, W-J. Dayanand, A. Tang, S-K. Tian, X. Zhi, X. Hua, L. Jiang, C. 2006. Seperation, identification and analysis of pigment (melanin) production in Streptomyces. African Journal of Biotechnology.; 5(8): 1131-1134.

10.Duran, B., Álzate, F. 2009. Intercepción de partículas suspendidas totales (PST) por cinco especies de árboles urbanos en el Valle de Aburrá. Revista Facultad de Ingeniería Universidad de Antioquia [online]. n.47 [citado 2014-04-29], pp. 59-66. Disponible en: <http://www.scielo.org.co/scielo.php?script= sci_arttext\&pid=S0120-62302009000100006\&lng= pt\&nrm=iso>. ISSN 0120-6230

11. Instituto Colombiano de Normas Técnicas y Certificación. 2005. Sistemas de gestión de inocuidad de los alimentos. Requisitos para cualquier organización en la cadena alimentaria. NTC-ISO 22000. Bogotá D.C.

12. Instituto Colombiano de Normas Técnicas y Certificación. 2009. Microbiología de alimentos y productos para alimentación animal. Requisitos generales y directrices para análisis microbiológicos. NTC 4092. Bogotá D.C.

13.León, J. 2011. Estudio investigativo de la “pomarrosa y su aplicación en la gastronomía. Trabajo de grado Administrador Gastronómico. Ecuador. Universidad Tecnológica Equinoccial. Carrera: Turismo y Preservación Ambiental, Hotelería y Gastronomía.

14.Libkind, D., Ruda, M., Andrade, J., Brizzio, S., Ruffini, A., Moline, M., De García, V., Russo, G. 2000. Biodiversidad y biotecnología de levaduras nativas de la región de la Patagonia. Laboratorio de microbiología aplicada y biotecnología y laboratorio de química orgánica CAB-CNEA. Buenos Aires. Disponible en: <http://www.uncoma.edu.ar/secinves tigacion/PRO_2005_POR_UA/CRUB/B121.htm>

15.Lindow, S., Hecht-Poinar, E., Elliott, V. 2004. Phyllosphere microbiology. American Phyto- 
pathological Society. United States of America. p. 393.

16. Mager, W., Siderius, M. 2002. Novel insights into Theosmotic stress response of yeast. FEMS Yeast Research. Volumen 2, pp. 251-257.

17.Medina, M., Calderón, E. 2008. Estudio anatómico del pomorroso (Sizygium malaccense (l). Merr. \& L.M Perry) y fotoquímico preliminar de su fruto en la comuna 1 de la ciudad de Neiva - Huila. Trabajo de grado Licenciado en Ciencias Naturales. Neiva. Universidad Surcolombiana. Facultad de Educación. Programa de Ciencias Naturales.

18.Panizo, M., Reviákina, V., Montes, W., González, G. 2005. Mantenimiento y preservación de hongos en agua destilada y aceite mineral. Revista de la So- ciedad Venezolana de Microbiología. [Online]. vol.25, n.1 [citado 2014-02-22], pp. 35-40. Disponible en: <http://www.scielo.org.ve/scielo.php? script=sci_arttext\&pid=S1315-25562005000100007 \&lng $=\mathrm{es} \& \mathrm{nrm}=\mathrm{iso}>$. ISSN 1315-2556

19.Uribe, L. 2007. Caracterización fisiológica de levaduras aisladas de la filósfera de la mora. Trabajo de grado Microbiólogo Industrial. Bogotá D.C. Pontificia Universidad Javeriana. Facultad de Ciencias.

20.Usman, L. 2012. Aislamiento, identificación molecular y caracterización parcial de levaduras asociadas a la pulpa de los frutos de mango (Mangifera indica) y pomorroso (Syzygium malaccense). Trabajo de grado Bióloga con mención en Microbiología. Santiago de Cali. Universidad del Valle. Facultad de Ciencias Naturales y Exactas. Programa de Biología. 
